\title{
Double Tracking Control for the Directed Complex Dynamic Network via the Observer of Outgoing Links
}

\section{Peitao Gao ( $\sim$ peitao_gao@sina.com )}

Guangdong University of Technology https://orcid.org/0000-0002-2538-2223

\section{Yinhe Wang}

Guangdong University of Technology

\section{Lizhi Liu}

Guangdong University of Technology

\section{Lili Zhang}

Guangdong University of Technology

\section{Xiao Tang}

Guangdong University of Technology

\section{Research Article}

Keywords: directed complex dynamic network, nodes subsystem, outgoing links subsystem, double tracking control

Posted Date: March 2nd, 2021

DOl: https://doi.org/10.21203/rs.3.rs-255359/v1

License: (c) (1) This work is licensed under a Creative Commons Attribution 4.0 International License. Read Full License 


\title{
Double Tracking Control for the Directed Complex Dynamic Network via the State Observer of Outgoing Links
}

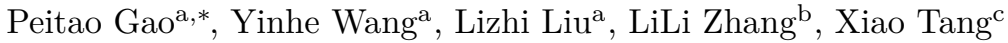 \\ ${ }^{a}$ School of Automation, Guangdong University of Technology, Guangzhou 510006, P.R. China \\ ${ }^{b}$ School of Applied Mathematics, Guangdong University of Technology, Guangzhou 510006, P.R. China \\ ${ }^{c}$ Zhongkeqidi Optoelectronic Technology(Guangzhou) Co., Ltd. Guangzhou, 510006, P.R. China
}

\begin{abstract}
From the large system perspective, the directed complex dynamic network is considered as being composed of the nodes subsystem (NS) and the links subsystem (LS), which are coupled with together. Different from the previous studies which propose the dynamic model of LS with the matrix differential equations, this paper describes the dynamic behavior of LS with the outgoing links vector at every node, by which the dynamic model of LS can be represented as the vector differential equation to form the outgoing links subsystem (OLS). Since the vectors possess the flexible mathematical operational properties than matrices, this paper proposes the more convenient mathematic method to investigate the double tracking control problems of NS and OLS. Under the state of OLS can be unavailable, the asymptotical state observer of OLS is designed in this paper, by which the tracking controllers of NS and OLS are synthesized to ensure achieving the double tracking goals. Finally, the example simulations for supporting the theoretical results are also provided.
\end{abstract}

Keywords: directed complex dynamic network, nodes subsystem, outgoing links subsystem, double tracking control.

\section{Introduction}

In the past twenty years, the complex dynamic network have been widely used in many real networks such as neural networks [1] 3], power networks [4], traffic networks [5] and wireless sensor networks[6, 7]. It is well known in graph theory that a complex dynamic network can be considered 5 as consisting of a group of dynamic nodes and the dynamic links that connect them. From the large system perspective, all the dynamic nodes can be regarded as the dynamic subsystem which is called as nodes subsystem (NS), and all the dynamic links can be regarded also as the dynamic subsystem which is called as links subsystem (LS), and thus the complex dynamic network consists of NS and LS coupled with each other [8 10]. By employing the above perspectives, the dynamic behaviors of the complex dynamic network can be reflected by the NS and the LS respectively. For

${ }^{*}$ Corresponding author at: School of Automation, Guangdong University of Technology Guangzhou 510006, P.R. China.

Email addresses: peitao_gao@sina.com (Peitao Gao), yinhewang@sina.com (Yinhe Wang), liulizhi_mingji9@sina.com (Lizhi Liu), zh_lili@sina.com (LiLi Zhang), zsu_tangxiao@hotmail.com (Xiao Tang) 
example, the synchronization 11 14] and stability 15] are regarded as the dynamic behavior of NS, and the asymptotic structural balance [16-18] is regarded as the dynamic behavior of LS.

It is worth noting that the above dynamic behaviors of NS and LS may be interpreted as the tracking problem in control theory. In other words, the synchronization can be interpreted as the NS tracking a reference state, and the asymptotic structural balance can be interpreted as the LS tracking a reference relation matrix. Corresponding to the above tracking problem, the "mutual assistance' plays an important role in the above literature, that is, through the mutual coupling relations between NS and LS, the tracking of NS is achieved with the help of LS, and the tracking of LS is achieved with the help of NS.

However, it is noticed that the tracking of NS and the tracking of LS are discussed separately in the above literature excepting [8, 9]. In [8, 9], the tracking targets of NS and LS are required to satisfy certain relation equalities, which limits the wide application. On the other hand, the weighted-values of links are defined as the state variables of LS in the above literature, and thus the dynamics of LS is represented as the Riccatti matrix differential (or difference in discrete-time) equations, by which the difficult theoretic analysis results in the complex mathematic conditions for the asymptotic structural balance.

It is also noticed in the above literature that it is assumed that the states of NS are measurable and the states of LS are not fully measurable. The states of LS are more complex and difficult to be measured than the states of NS in the complex dynamic network, so that it is necessary to employ the measurable outputs of LS to achieve the estimation of uncertain state variables for LS. At present, some research results have been achieved on the state observer [9, 19 21] design of LS. In [19], the state observer of LS is synthesized for the first time for the complex dynamic network. In [20, 21], the state observer employs the output of LS as its input to obtain the state observation information for LS, and then the LS tracks on a reference relation matrix by utilizing the state observation information to synthesize the controller of LS, without considering the dynamic behavior of NS. In view of this, the results in [9] extend the continuous state observer to the discrete state observer for LS. The LS tracks on a reference relation matrix via employing the controller which is designed by using the observation state information about LS. Nevertheless, the previous published studies have not dealt with the double tracking control problems of NS and LS.

Inspired by the above discussion, this paper focuses on the dynamics of the outgoing links at a node instead of the dynamics of overall links for all the nodes. In other words, this paper proposes that the dynamics of the outgoing links subsystem(OLS) is presented by all the outgoing links vectors(OLV) instead of the overall links matrix, where an OLV at a node is defined as the vector composed of all the weighted-values for the outgoing links at the node. Therefore, compared with the existing literature, the main innovations and contributions of this paper are as follows. (i) The dynamics of LS can be generated by the vector differential equation with the outgoing links, which is shown more simpler in structure than the matrix differential equations as in [8, 10, 16, 19 21]. This may reduce the difficulty of analysis due to vectors have more flexible mathematical operational properties than matrices [22, 23]. (ii) From the angle of state space in control theory, the OLV can be regarded as the state vector of outgoing links at a node, and thus it has more intuitive geometric feature than the overall link matrix. This implies that the dynamic model of OLS is more 
easily generalized to nonlinear situations with the help of those methods in control theory. (iii) By employing the state observer of outgoing links, the double tracking controllers of NS and OLS are designed separately to achieve the double tracking goals for the directed complex dynamic networks. That is, the NS tracks on the given target orbit when the state of OLS tracks the designed goal.

The remaining part of the paper proceeds as follows. In Section 2, the dynamic models of NS and OLS are proposed, and the directed characteristics of OLS are explained. The state observer for OLS is proposed in Section 3. In Section 4, by employing the observation information of OLS, the tracking controllers of NS and OLS are synthesized separately, by which the complex dynamic network can achieve the double tracking goals with some assumptions and definitions. Some numerical examples are given for supporting the theory results in Section 5. Finally, the conclusion is given in Section 6.

\section{Model description}

In this paper, we consider a directed time-varying complex dynamic network with $N$ nodes, the weighted-value of the $i$ th node pointing to the $j$ th node is represented as $l_{i j}=l_{i j}(t)$, where $l_{i j}(t)$ is treated as the self-link, $i, j=1,2, \cdots, N$. The dynamic equation of the $i$ th node is described as

$$
\dot{x}_{i}=A_{i} x_{i}+f_{i}\left(x_{i}\right)+c \sum_{k=1}^{N} l_{i k} \Lambda h_{k}(x)+u_{i},
$$

where $x_{i}=\left[x_{i 1}, x_{i 2}, \ldots, x_{i n}\right]^{T} \in R^{n}$ is the state vector of the $i$ th node, $x=\left[x_{1}^{T}, x_{2}^{T}, \ldots, x_{N}^{T}\right]^{T} \in R^{n N}$ is the state vector of NS, $A_{i} \in R^{n \times n}$ denotes the real constant matrix, $f_{i}\left(x_{i}\right)=\left[f_{i 1}\left(x_{i}\right), f_{i 2}\left(x_{i}\right), \ldots\right.$, $\left.f_{\text {in }}\left(x_{i}\right)\right]^{T}$ stands the nonlinear continuous vector function, $\Lambda=\operatorname{diag}\left(g_{1}, g_{2}, \ldots, g_{n}\right) \in R^{n \times n}$ is the diagonal matrix with the real constant $g_{l}, l=1,2, \ldots, n, h_{k}(x) \in R^{n}$ is the nonlinear smooth inner connection vector function. $c$ is the common coupling connection strength, and $u_{i} \in R^{n}$ denotes the control input, $i, k=1,2, \ldots, N$.

Inspired by the outgoing degree and incoming degree in the directed weighted networks [24], the following concepts are proposed.

Definition 1. $L_{i}(t)=\left[l_{i 1}(t), l_{i 2}(t), \ldots, l_{i N}(t)\right]^{T} \in R^{N}$ is called as the outgoing links vector of the $i$ th node. Similarly, the incoming links vector of the $i$ th node is defined as $\tilde{L}_{i}(t)=$ ${ }_{75}\left[l_{1 i}(t), l_{2 i}(t), \ldots, l_{N i}(t)\right]^{T}, i=1,2, \cdots, N$.

The dynamical equation (1) can be rewritten by the vector differential equation

$$
\dot{x}_{i}=A_{i} x_{i}+f_{i}\left(x_{i}\right)+c \Lambda H(x) L_{i}(t)+u_{i},
$$

where $H(x)=\left[h_{1}(x), h_{2}(x), \ldots, h_{N}(x)\right] \in R^{n \times N}, L_{i}(t)=\left[l_{i 1}(t), l_{i 2}(t), \ldots, l_{i N}(t)\right]^{T} \in R^{N}, i=$ $1,2, \cdots, N$.

Remark 1. (i) In Definition 1, the outgoing links vector at a node is utilized to describe the directional relations of a node pointing to the other nodes. This is inspired by the ones in the ego-network 25]. (ii) As part of the coupling, the outgoing links vector $L_{i}(t)$ is shown in Equation 
(2), which reflects the influence of the outgoing links of the $i$ th node on its dynamics. (iii) Noticing the relation matrix $\mathrm{Z}=Z(t)=\left[l_{i j}\right]_{N \times N}$, it is easily seen that the outgoing links vector $L_{i}(t)$ is the

$i$ th row of $Z(t)$, and the incoming links vector $\tilde{L}_{i}(t)$ is the $i$ th column of $Z(t), i=1,2, \cdots, N$. (iv) Especially, if $L_{i}(t)=\tilde{L}_{i}(t)$, the network is undirected.

The dynamical equation of the outgoing links subsystem (OLS) is regarded as follows

$$
\left\{\begin{array}{l}
\dot{L}_{i}=B_{i} L_{i}+\Phi_{i}(x)+U_{i}, \\
Y_{i}=C_{i} L_{i}
\end{array}\right.
$$

where $B_{i} \in R^{N \times N}$ is the real constant matrix, $C_{i} \in R^{N_{1} \times N}$ is the output matrix, $\Phi_{i}(x) \in R^{N}$ is the coupling vector, $U_{i} \in R^{N}$ stands the control input, $Y_{i} \in R^{N_{1}}$ denotes the output of OLS, 90 $i=1,2, \cdots, N$.

Remark 2. (i) Equation (3) represents the outgoing links vector dynamics of the $i$ th node, in which the vector $\Phi_{i}(x)$ shows the coupling relationship between the outgoing links vector of the $i$ th node and the state of all the nodes. (ii) The vector representation (3) for LS is relatively rare in the existing literature on the observer design for LS, which helps to reduce the difficulty of theoretical analysis because of the vectors possessing more flexible mathematical operational properties than matrices. (iii) Equation (3) (without controller $U_{i}$ ) can be rewritten as follows

$$
\left\{\begin{aligned}
i_{i j} & =\sum_{k=1}^{N} b_{j k}^{i} l_{i k}+\psi_{j}^{i}(x), \\
y_{s}^{i} & =\sum_{k=1}^{N} c_{s k}^{i} l_{i k},
\end{aligned}\right.
$$

where $B_{i}=\left(b_{j k}^{i}\right)_{N \times N}$ is the real constant matrix, $\Phi_{i}=\left(\psi_{j}^{i}(x)\right) \in R^{N}$ is the coupling vector, $Y_{i}=\left(y_{s}^{i}\right)_{N_{1} \times 1}$ denotes the output of the outgoing links vector, $C_{i}=\left(c_{s k}^{i}\right)_{N_{1} \times N}$ stands the output matrix, $i, j, k=1,2, \cdots, N, s=1,2, \cdots, N_{1}$. The schematic diagram of Equation (4) may be explained as follows

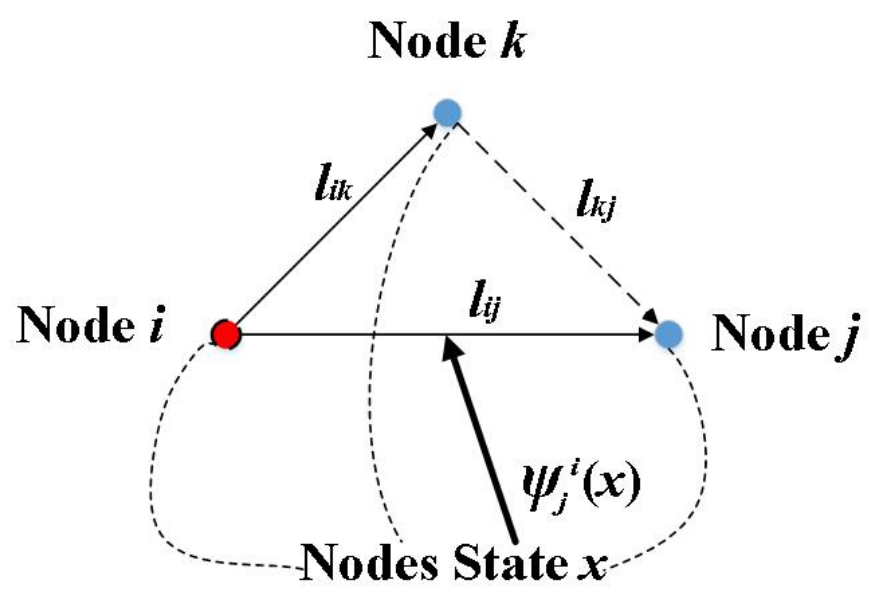

Fig.1 The schematic diagram of $l_{i j}$ influenced by $l_{i k}$ and the coupling vector $\psi_{j}^{i}(x)$ in Equation (4) 
In Fig.1, the node $k$ represents the node other than node $i$ and $j$. Fig.1 means that the time derivative of $l_{i j}$ can be influenced directly by the linear combination operation of $l_{i k}$ s for all $1 \leq$ $k \leq N$ with the coupling term $\psi_{j}^{i}(x)$. This linear combination is regarded as the approximation linearization of nonlinear function with the arguments $l_{i k} \mathrm{~s}, i, j, k=1,2, \cdots, N$.

\section{Asymptotical state observer of the outgoing links subsystem}

Definition 2. Consider the complex dynamic networks with (2) and (3). If the state of NS in (2) can be measured exactly and there exists the dynamical system $\dot{\hat{L}}_{i}=F_{i}\left(\hat{L}_{i}, Y_{i}, x\right)$ such that ${ }_{110} E_{i}=L_{i}-\hat{L}_{i} \stackrel{t \rightarrow+\infty}{\longrightarrow} 0$ holds, then the dynamical system $\dot{\hat{L}}_{i}=F_{i}\left(\hat{L}_{i}, Y_{i}, x\right)$ is called as the $i$ th asymptotical state observer of the $i$ th OLS, $i=1,2, \cdots, N$.

According to Definition 2, the $i$ th asymptotical state observer of the $i$ th OLS is as follows

$$
\dot{\hat{L}}_{i}=B_{i} \hat{L}_{i}+\Phi_{i}(x)-K_{i}\left(Y_{i}-C_{i} \hat{L}_{i}\right)+U_{i}
$$

where $K_{i} \in R^{N \times N_{1}}$ is the matrix of coefficients to be determined, $i=1,2, \cdots, N$.

Assumption 1. Consider the OLS (3). The double matrices $\left(B_{i}, C_{i}\right)$ is completely observable.

If Assumption 1 holds, there exists a matrix $K_{i}$ with suitable dimensions, which can make $B_{i}+K_{i} C_{i}$ to be a Hurwitz stable matrix, and thus there exists only one positive definition matrix $M_{i} \in R^{N \times N}$ satisfying the following Lyapunov equation for the given positive definition matrix $Q_{i}$, $i=1,2, \cdots, N$.

$$
\left(B_{i}+K_{i} C_{i}\right)^{T} M_{i}+M_{i}\left(B_{i}+K_{i} C_{i}\right)=-Q_{i}
$$

Remark 3. In Lyapunov equation (6), the matrices $K_{i}$ and $M_{i}$ can be obtained by solving the linear matrix inequality $W_{1} B_{i}^{T}+B_{i} W_{1}+C_{i} W_{2}+W_{2}^{T} C_{i}^{T}<0$, where $W_{1}>0, W_{1}=K_{i}^{-1}$, $W_{2}=M_{i} W_{1}$. The details for obtaining the above solution can be referred to the solving steps in $[26,27]$.

Lemma 1. If Assumption 1 holds, then Equation (5) is the asymptotical state observer for OLS (3).

125 Proof 1. Let the observation error $E_{i}=L_{i}-\hat{L}_{i}$, then the following equation can be obtained, $i=1,2, \cdots, N$.

$$
\begin{aligned}
\dot{E}_{i} & =\dot{L}_{i}-\dot{\hat{L}}_{i} \\
& =B_{i} L_{i}+\Phi_{i}(x)+U_{i}-\left[B_{i} \hat{L}_{i}+\Phi_{i}(x)-K_{i}\left(Y_{i}-C_{i} \hat{L}_{i}\right)+U_{i}\right] \\
& =B_{i}\left(L_{i}-\hat{L}_{i}\right)+K_{i}\left(C_{i} L_{i}-C_{i} \hat{L}_{i}\right) \\
& =\left(B_{i}+K_{i} C_{i}\right) E_{i} .
\end{aligned}
$$

Noticing that $B_{i}+K_{i} C_{i}$ is the Hurwitz stable matrix due to Assumption 1, it is seen that $E_{i}$ is bounded and $E_{i}=L_{i}-\hat{L}_{i} \stackrel{t \rightarrow+\infty}{\longrightarrow} 0$. Lemma 1 is proved.

By using Lemma 1, the control input $U_{i}$ in OLS (3) can be synthesized by employing the state in Equations (5) and (2), which helps to synthesize the double tracking control scheme for NS and OLS. 


\section{Double tracking control design based on observer}

Let $x_{i}^{*}=x_{i}^{*}(t) \in R^{n}$ and $L_{i}^{*}=L_{i}^{*}(t) \in R^{N}$ are chosen as the state reference tracking goals of NS and OLS, respectively, and suppose that they are the differentiable bounded, $i=1,2, \cdots, N$.

Definition 3. Consider the directed complex dynamic network with (1) and (3). If $\lim _{t \rightarrow \infty}\left(L_{i}-\right.$ $\left.L_{i}^{*}\right)=0$ and $\lim _{t \rightarrow \infty}\left(x_{i}-x_{i}^{*}\right)=0$ hold for all $i=1,2, \cdots, N$, the directed complex dynamic network is called as achieving the double tracking goals.

Control objective: Suppose that the state $x_{i}$ of NS are available and $L_{i}$ are unavailable for $i=1,2, \cdots, N$. By employing the state of observer (5), synthesize the controller $u_{i}$ for NS (2) and the controller $U_{i}$ for OLS (3), respectively, such that the complex dynamic network can achieve the double tracking goals.

Remark 4. The control objective of this paper is largely inspired by [28 30]. he web processing line may be treated to be composed of the roller (nodes) and the coupled web (the outgoing links), in which the roller velocity $s_{i}$ and web tension $T_{i}$ are regarded as the state variables of NS and OLS, respectively. Let $\bar{T}_{i}=T_{i}-T_{r i}$ and $\bar{s}_{i}=s_{i}-s_{r i}$, where $T_{r i}$ and $s_{r i}$ are the reference tension and velocity, respectively. It can be observed that the control objective for the web processing line is consistent with the one in this paper.

From Assumption 2, it is seen that the following Lyapunov equation has only one positive definition matrix solution $P_{i} \in R^{n \times n}$ for the given positive definition matrices $\tilde{Q}_{i}, i=1,2, \cdots, N$.

$$
\left(A_{i}+\bar{K}_{i}\right)^{T} P_{i}+P_{i}\left(A_{i}+\bar{K}_{i}\right)=-\tilde{Q}_{i},
$$

Remark 5. In Lyapunov equation (8), the matrices $\bar{K}_{i}$ and $P_{i}$ can be obtained by solving the linear matrix inequality $A_{i}^{T} P_{i}+P_{i} A_{i}+W_{4}+W_{4}^{T}<0$, where $P_{i}>0, W_{4}=P_{i} \bar{K}_{i}$.

Assumption 2. Consider the NS (2). There exists a matrix $\bar{K}_{i} \in R^{n \times n}$ such that $A_{i}+$ $\bar{K}_{i}$ is a Hurwitz matrix, the nonlinear vector function $f_{i}\left(x_{i}\right)$ is bounded, that is, $\left\|f_{i}\left(x_{i}\right)\right\| \leq$ $\eta_{i}\left(x_{i}\right)$, where $\eta_{i}\left(x_{i}\right) \geq 0$ is a known function, and the inner connection function meets $\|H(x)\|<$

$\min _{\leq i \leq N}\left\{\frac{\lambda_{\min }\left(\tilde{Q}_{i}\right)}{\sigma c\|\Lambda\|\left\|P_{i}\right\|}, \frac{\lambda_{\min }\left(Q_{i}\right) \sigma}{c\|\Lambda\|\left\|P_{i}\right\|}\right\}$ and $0<\sigma<\lambda_{\min }\left(\tilde{Q}_{i}\right)$, where $\sigma$ is an adjustable positive parameter. $\|*\|$ stands the Euclidean norm of the matrix or the vector ' $*$ '. $\lambda_{\min }(*)$ represents the minimum eigenvalue of matrix ' $*$ ', $i=1,2, \cdots, N$.

Assumption 3. Consider the OLS (3). The coupling function $\Phi_{i}(x)$ is bounded, that is, $\left\|\Phi_{i}(x)\right\| \leq g_{i}(x)$, where $g_{i}(x) \geq 0$ is a known function, $i=1,2, \cdots, N$.

Based on the above control objective, the following double tracking controller $u_{i}$ and $U_{i}$ are proposed for NS (2) and OLS (3), respectively.

$$
\begin{gathered}
u_{i}=\bar{K}_{i} x_{i}+\dot{x}_{i}^{*}-\left(A_{i}+\bar{K}_{i}\right) x_{i}^{*}+c \Lambda H(x) \hat{L}_{i}+v_{i}, \\
v_{i}=\left\{\begin{array}{cc}
-\frac{P_{i} e_{i}}{\left\|e_{i}^{T} P_{i}\right\|} \eta_{i}\left(x_{i}\right), & e_{i} \neq 0, \\
0, & e_{i}=0, \\
U_{i}=K_{i} Y_{i}-\left(B_{i}+K_{i} C_{i}\right) L_{i}^{*}+\dot{L}_{i}^{*}+\bar{V}_{i},
\end{array}\right.
\end{gathered}
$$




$$
\bar{V}_{i}=\left\{\begin{array}{cc}
-\frac{M_{i} \widehat{E}_{i}}{\left\|\widehat{E}_{i}^{T} M_{i}\right\|} g_{i}(x), & \widehat{E}_{i} \neq 0 . \\
0, & \widehat{E}_{i}=0 .
\end{array}\right.
$$

Let the tracking errors $e_{i}=x_{i}-x_{i}^{*}$ and $\widehat{E}_{i}=\hat{L}_{i}-L_{i}^{*}$. Consider the network with (2) and (3), by using the controllers (9) and (11), the error dynamical equations are obtained as follows

$$
\begin{aligned}
\dot{e}_{i}= & \dot{x}_{i}-\dot{x}_{i}^{*} \\
= & A_{i} x_{i}+f_{i}\left(x_{i}\right)+c \Lambda H(x) L_{i}+u_{i}-\dot{x}_{i}^{*} \\
= & \left(A_{i}+\bar{K}_{i}\right)\left(x_{i}-x_{i}^{*}\right)+c \Lambda H(x)\left(L_{i}-\hat{L}_{i}\right)+f_{i}\left(x_{i}\right)+v_{i} \\
= & \left(A_{i}+\bar{K}_{i}\right) e_{i}+c \Lambda H(x) E_{i}+f_{i}\left(x_{i}\right)+v_{i}, \\
& \widehat{E}_{i}=\dot{\hat{L}}_{i}-\dot{L}_{i}^{*} \\
& =B_{i} \hat{L}_{i}+\Phi_{i}(x)-K_{i}\left(Y_{i}-C_{i} \hat{L}_{i}\right)+U_{i}-\dot{L}_{i}^{*} \\
& =B_{i} \hat{L}_{i}+K_{i} C_{i} \hat{L}_{i}-K_{i} Y_{i}+\Phi_{i}(x)+U_{i}-\dot{L}_{i}^{*} \\
& =\left(B_{i}+K_{i} C_{i}\right) \widehat{E}_{i}+\Phi_{i}(x)+\bar{V}_{i} .
\end{aligned}
$$

Considering the positive definite function $V_{1}=V_{1}(t)=\sum_{i=1}^{N} e_{i}^{T} P_{i} e_{i}+\sum_{i=1}^{N} \widehat{E}_{i}^{T} M_{i} \widehat{E}_{i}+\sum_{i=1}^{N} E_{i}^{T} M_{i} E_{i}$, where $P_{i} \in R^{n \times n}$ and $M_{i} \in R^{N \times N}$ are determined by (8) and (6), respectively. Let $d_{1}=\lambda_{\min }\left(\tilde{Q}_{i}\right)-$ $c\left\|[\Lambda H(x)]^{T} P_{i}\right\| \sigma, d_{2}=\lambda_{\min }\left(Q_{i}\right)-\frac{c\left\|[\Lambda H(x)]^{T} P_{i}\right\|}{\sigma}$ and $d_{3}=\lambda_{\min }\left(Q_{i}\right)$. If Assumptions 1-3 are satisfied, by employing the controllers (10) and (12), the orbit derivative of $V_{1}=V_{1}(t)$ along the error systems (13) and (14) is obtained Equation (15), (16) and inequality (17).

$$
\begin{aligned}
\dot{V}_{1} & =\sum_{i=1}^{N} \dot{e}_{i}^{T} P_{i} e_{i}+\sum_{i=1}^{N} e_{i}^{T} P_{i} \dot{e}_{i}+\sum_{i=1}^{N} \dot{\vec{E}}_{i}^{T} M_{i} \widehat{E}_{i}+\sum_{i=1}^{N} \widehat{E}_{i}^{T} M_{i} \dot{\widehat{E}}_{i}+\sum_{i=1}^{N} \dot{E}_{i}^{T} M_{i} E_{i}+\sum_{i=1}^{N} E_{i}^{T} M_{i} \dot{E}_{i} \\
& =\sum_{i=1}^{N}\left[\left(A_{i}+\bar{K}_{i}\right) e_{i}+c \Lambda H(x) E_{i}+f_{i}\left(x_{i}\right)+v_{i}\right]^{T} P_{i} e_{i} \\
& +\sum_{i=1}^{N} e_{i}^{T} P_{i}\left[\left(A_{i}+\bar{K}_{i}\right) e_{i}+c \Lambda H(x) E_{i}+f_{i}\left(x_{i}\right)+v_{i}\right] \\
& +\sum_{i=1}^{N}\left[\left(B_{i}+K_{i} C_{i}\right) \widehat{E}_{i}+\Phi_{i}(x)+\bar{V}_{i}\right]^{T} M_{i} \widehat{E}_{i}+\sum_{i=1}^{N} \widehat{E}_{i}^{T} M_{i}\left[\left(B_{i}+K_{i} C_{i}\right) \widehat{E}_{i}+\Phi_{i}(x)+\bar{V}_{i}\right] \\
& +\sum_{i=1}^{N}\left[\left(B_{i}+K_{i} C_{i}\right) E_{i}\right]^{T} M_{i} E_{i}+\sum_{i=1}^{N} E_{i}^{T} M_{i}\left(B_{i}+K_{i} C_{i}\right) E_{i},
\end{aligned}
$$




$$
\begin{aligned}
& \dot{V}_{1}=\sum_{i=1}^{N} e_{i}^{T}\left(A_{i}+\bar{K}_{i}\right)^{T} P_{i} e_{i}+\sum_{i=1}^{N} e_{i}^{T} P_{i}\left(A_{i}+\bar{K}_{i}\right) e_{i} \\
& +\sum_{i=1}^{N} \widehat{E}_{i}^{T}\left(B_{i}+K_{i} C_{i}\right)^{T} M_{i} \widehat{E}_{i}+\sum_{i=1}^{N} \widehat{E}_{i}^{T} M_{i}\left(B_{i}+K_{i} C_{i}\right) \widehat{E}_{i} \\
& +\sum_{i=1}^{N} E_{i}^{T}\left(B_{i}+K_{i} C_{i}\right)^{T} M_{i} E_{i}+\sum_{i=1}^{N} E_{i}^{T} M_{i}\left(B_{i}+K_{i} C_{i}\right) E_{i} \\
& +\sum_{i=1}^{N} 2 c E_{i}^{T}[\Lambda H(x)]^{T} P_{i} e_{i}+2 \sum_{i=1}^{N} \widehat{E}_{i}^{T} M_{i}\left[\Phi_{i}(x)+\bar{V}_{i}\right]+2 \sum_{i=1}^{N} e_{i}^{T} P_{i}\left[f_{i}\left(x_{i}\right)+v_{i}\right] \\
& \dot{V}_{1} \leq-\sum_{i=1}^{N} e_{i}^{T} \tilde{Q}_{i} e_{i}-\sum_{i=1}^{N} \widehat{E}_{i}^{T} Q_{i} \widehat{E}_{i}-\sum_{i=1}^{N} E_{i}^{T} Q_{i} E_{i}+\sum_{i=1}^{N} 2 c E_{i}^{T}[\Lambda H(x)]^{T} P_{i} e_{i} \\
& \leq-\lambda_{\min }\left(\tilde{Q}_{i}\right)\left\|e_{i}\right\|^{2}-\lambda_{\min }\left(Q_{i}\right)\left\|\widehat{E}_{i}\right\|^{2}-\lambda_{\min }\left(Q_{i}\right)\left\|E_{i}\right\|^{2} \\
& +2 c\left\|E_{i}\right\|\left\|[\Lambda H(x)]^{T} P_{i}\right\|\left\|e_{i}\right\| \\
& \leq-\lambda_{\min }\left(\tilde{Q}_{i}\right)\left\|e_{i}\right\|^{2}-\lambda_{\min }\left(Q_{i}\right)\left\|\widehat{E}_{i}\right\|^{2}-\lambda_{\min }\left(Q_{i}\right)\left\|E_{i}\right\|^{2} \\
& +c\left\|[\Lambda H(x)]^{T} P_{i}\right\|\left(\frac{1}{\sigma}\left\|E_{i}\right\|^{2}+\sigma\left\|e_{i}\right\|^{2}\right) \\
& =-\left(\lambda_{\min }\left(\tilde{Q}_{i}\right)-c\left\|[\Lambda H(x)]^{T} P_{i}\right\| \sigma\right)\left\|e_{i}\right\|^{2} \\
& -\left(\lambda_{\min }\left(Q_{i}\right)-\frac{c\left\|[\Lambda H(x)]^{T} P_{i}\right\|}{\sigma}\right)\left\|E_{i}\right\|^{2}-\lambda_{\min }\left(Q_{i}\right)\left\|\widehat{E}_{i}\right\|^{2} \\
& =-\left(\begin{array}{c}
\left\|e_{i}\right\| \\
\left\|E_{i}\right\| \\
\left\|\widehat{E}_{i}\right\|
\end{array}\right)^{T}\left(\begin{array}{ccc}
d_{1} & 0 & 0 \\
0 & d_{2} & 0 \\
0 & 0 & d_{3}
\end{array}\right)\left(\begin{array}{c}
\left\|e_{i}\right\| \\
\left\|E_{i}\right\| \\
\left\|\widehat{E}_{i}\right\|
\end{array}\right) \leq 0 .
\end{aligned}
$$

The inequality (17) means that $\dot{V}_{1}$ is the negative definite function about $e_{i}, E_{i}$ and $\widehat{E}_{i}$, and thus the error system with (13) and (14) is asymptotically stable, this is, $\lim _{t \rightarrow \infty}\left(x_{i}-x_{i}^{*}\right)=0$, $\lim _{t \rightarrow \infty}\left(L_{i}-\hat{L}_{i}\right)=0$ and $\lim _{t \rightarrow \infty}\left(\hat{L}_{i}-L_{i}^{*}\right)=0$, which implies $\lim _{t \rightarrow \infty}\left(L_{i}-L_{i}^{*}\right)=0$. Therefore, the complex dynamic network can achieve the double tracking goals which is shown in Theorem 1.

Theorem 1. Consider the directed complex dynamic network with (2), (3) and the state observer (5) for the OLS (3). If Assumptions 1-3 are satisfied, the complex dynamic network can achieve the double tracking goals.

Theorem 6. For applying Theorem 1 to achieve the double tracking control of the complex dynamic network, the steps are proposed as follows

Step 1. Giving the differential bounded vector signals $x_{i}^{*}(t)$ and $L_{i}^{*}$.

Step 2. Utilizing the output of OLS to design its observer (5), and the determined matrices $K_{i}$, $M_{i}, P_{i}$ and $\bar{K}_{i}$ can be obtained by solving the linear matrix inequality in Remark 3 and Remark 5 , respectively. 
Step 3. By using the observed information of OLS from Step2, the controller (9)-(10) of the NS and the controller (11)-(12) of OLS are designed separately.

Step 4. By employing the controllers (9)-(12) of NS and OLS from Step3, the complex dynamic $g_{4}$ are randomly generated in the interval $[-4,4]$, the control input $u_{i}$ is given as (9)-(10); Furthermore, $H(x)=\frac{1-e^{-x}}{1+e^{-x}}$ denotes the inner connection vector function. It is obvious that the matrix $A_{i}$ is a Hurwitz stable matrix. Moreover, let the common coupling strength $c$ is randomly generated in the interval $[0,1]$.

The generation rules of matrices $B_{i}, K_{i}, \bar{K}_{i}, P_{i}, M_{i}, C_{i}$ and the double tracking goals $x_{i}^{*}, L_{i}^{*}$, the initial state values $x_{i}^{*}(0), L_{i}^{*}(0), \hat{L}_{i}(0)$ are as follows according to the command statement 'randn' in Matlab.

(i) Let $B_{i}=m_{1} * \operatorname{randn}(N, N), \bar{K}_{i}=m_{2} * \operatorname{randn}(4 N, 4 N)$ and $C_{i}=m_{3} * \operatorname{randn}(10, N)$, where $m_{1}, m_{2}$ and $m_{3}$ are adjustable parameters, in this simulation, $m_{1}=m_{2}=m_{3}=8$.

(ii) Solving for the positive definite matrices $M_{i}$ and $K_{i}$ such that linear matrix inequality $W_{1} B_{i}^{T}+B_{i} W_{1}+C_{i} W_{2}+W_{2}^{T} C_{i}^{T}<0$, where $W_{1}>0$ holds, the observed gain matrix $M_{i}=W_{2} K_{i}^{-1}$ is a Hurwitz stable matrix, where $K_{i}=W_{1}^{-1}$. Similarly, solving for the positive definite matrix $P_{i}$ 
such that linear matrix inequality $A_{i}^{T} P_{i}+P_{i} A_{i}+W_{4}+W_{4}^{T}<0$ holds, the $P_{i}=W_{4} \bar{K}_{i}^{-1}$ is a Hurwitz stable matrix.

(iii) Let the double tracking goals $x_{i}^{*}=[\theta \sin (t), \theta \cos (t), \theta \tan (t), \theta \tanh (t)]^{T}$ and $L_{i}^{*}=i \theta \sin (t)$, where $\theta$ is an adjustable parameter, and its value is randomly generated real numbers in the interval $[-8,8], i=1,2, \cdots, N$.

(iv) The initial state values of NS and OLS are $x_{i}(0)=\operatorname{randn}(4 N, 1)$ and $L_{i}(0)=\operatorname{randn}(N, 1)$, respectively. Moreover, the initial state values of observer $\hat{L}_{i}(0)=(N, 1)$.

The dynamics of $L_{i}(t)$ is chosen as (3). The coupling term $\Phi_{i}(x)$ meets Assumption 3, the control input $U_{i}$ are given as (11)-(12). In the process of simulation, the other parameters are selected as follows. $N=30, \sigma=\operatorname{rand}(1)$ is an adjustable parameter, $\tilde{Q}_{i}=I_{i}$, where $I_{i}$ is 4-dimensional unit matrix. In order to show the advantage of this paper, the simulation results in this paper are compared with the results in $\left[19\right.$ 21], respectively. By using the norm $\|\vartheta\|=\sqrt{\sum_{k}^{m}\left|\vartheta_{k}\right|^{2}}$ for the real vector $\vartheta=\left(\vartheta_{1}, \vartheta_{2}, \ldots, \vartheta_{m}\right)^{T} \in R^{m}$, the compared simulation results are shown in Figs.2-5 as follows.
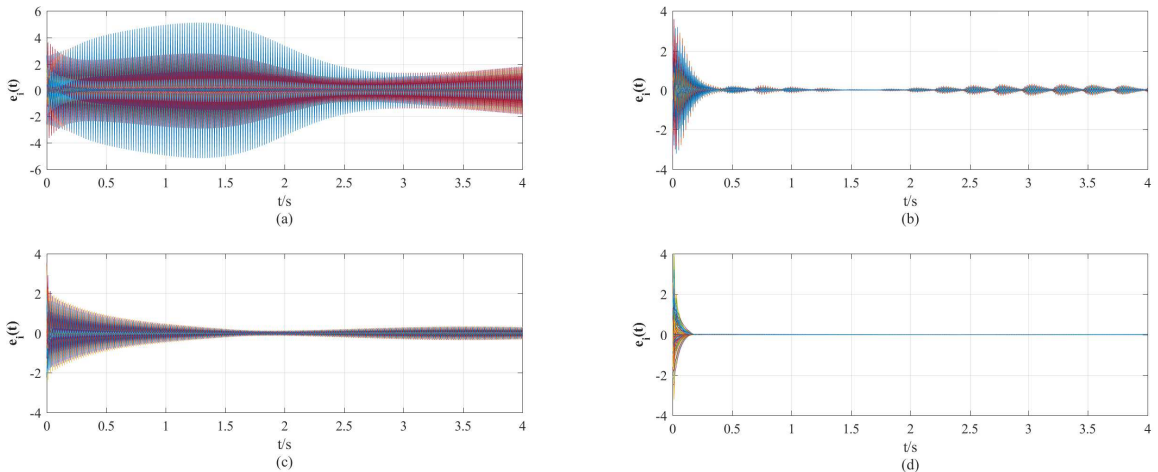

Fig.2 (a).The tracking error curves of NS with the controller in [19]; (b). The tracking error curves of NS with the controller in [20]; (c). The tracking error curves of NS with the controller in [21]; (d). The tracking error curves of NS with the controller in this paper
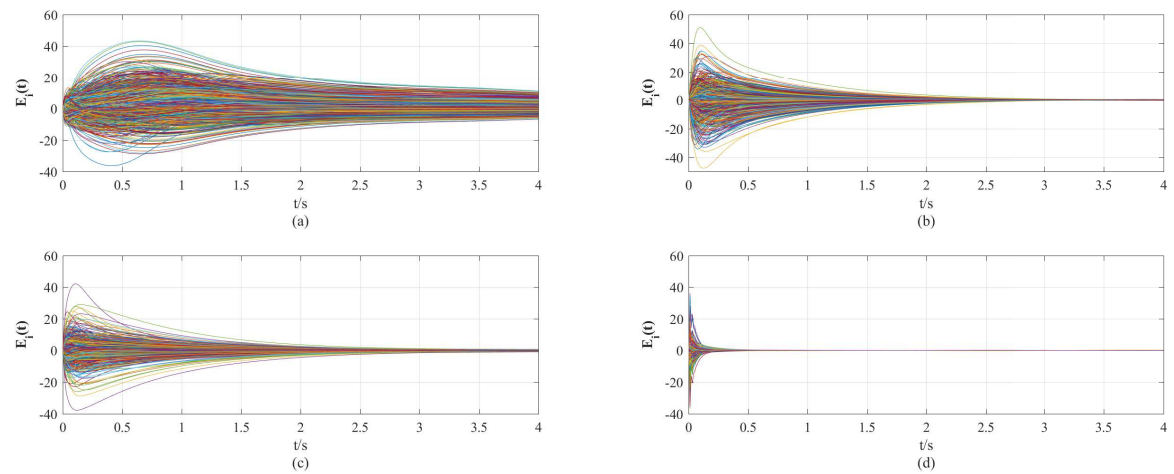

Fig.3 (a).The tracking error curves of OLS with the controller in [19]; (b). The tracking error curves of

OLS with the controller in [20]; (c). The tracking error curves of OLS with the controller in [21]; (d).The tracking error curves of OLS with the controller in this paper 

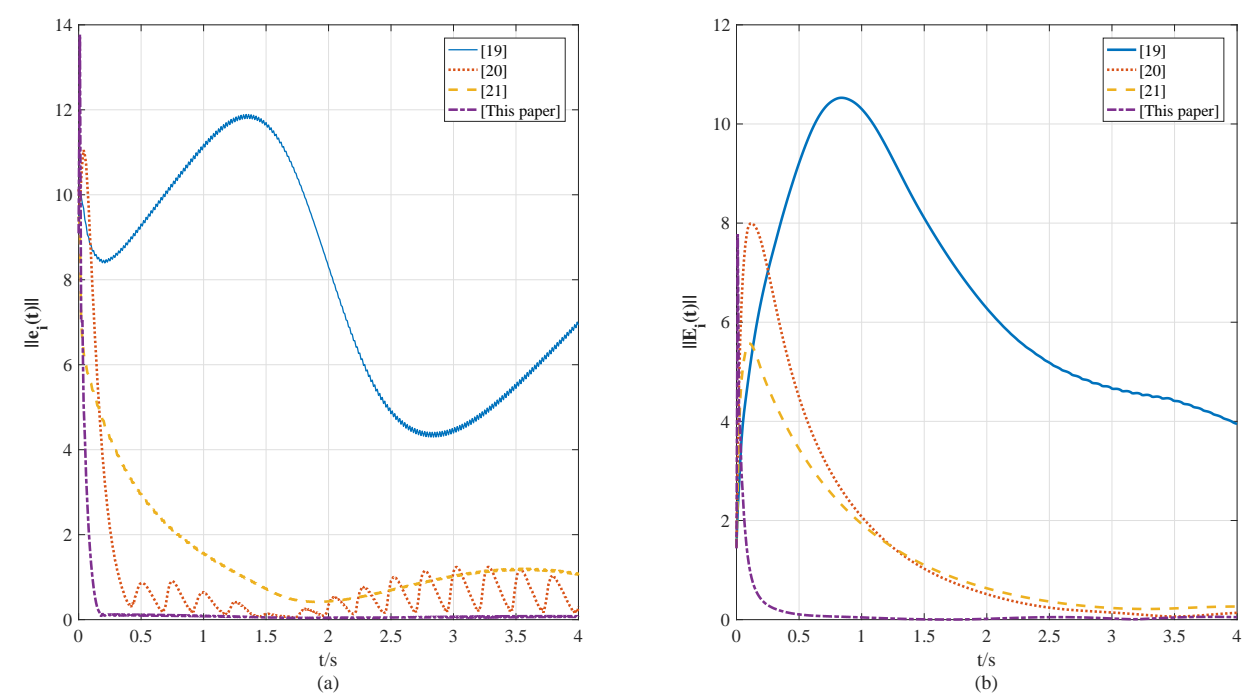

Fig.4 (a).The curves of tracking error norm $\left\|e_{i}\right\|$ of NS with the controller in [19 21] and this paper;

(b). The curves of tracking error norm $\left\|E_{i}\right\|$ of OLS with the controller in 19 21] and this paper

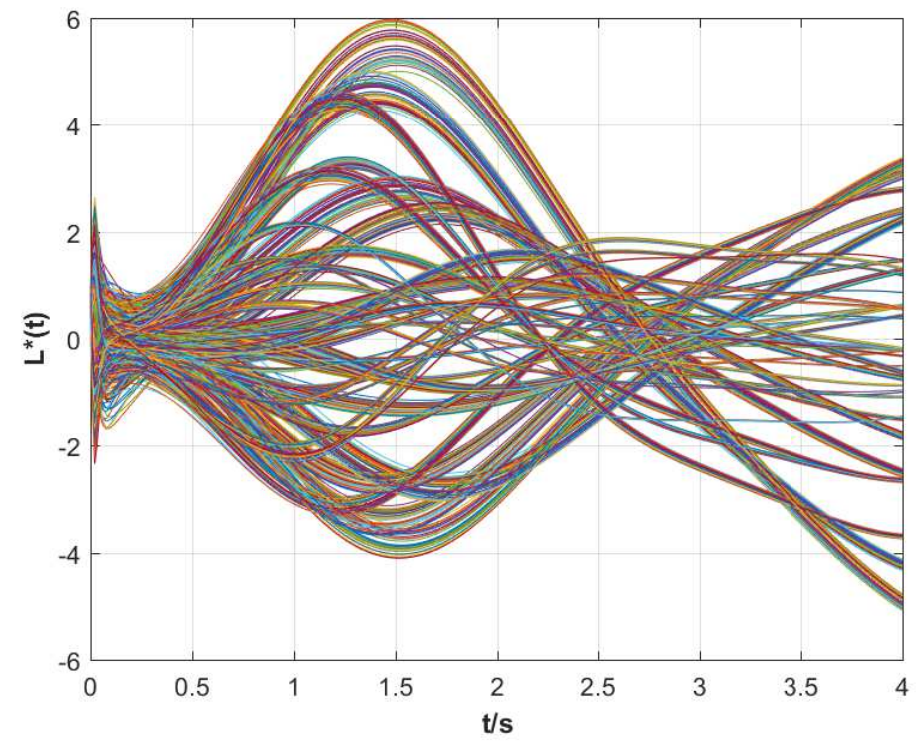

Fig.5 The orbit curves of $L_{i}^{*} \mathrm{~s}$ in this paper

From Figs.2-5, we can obtain the following conclusion.

(i) From Fig.2 and Fig.4, it can be seen that the tracking controllers in [19 21] are designed by utilizing the observer's state of LS to control NS to track the reference state, but tracking error curves show not only the larger chattering problem but also the slower convergence speeds than ones in this paper. Similarly, it can be observed from Fig.3 and Fig.4 that the tracking controllers are synthesize by employing the observer's sate of LS to control LS to track the differentiable bounded reference goal, but the tracking response speeds of OLS in [19 21] are also slower than that in this 
paper.

(ii) By utilizing the norms $\left\|e_{i}\right\|$ and $\left\|E_{i}\right\|$ in Fig.4, where $E_{i}$ represents the error between $L_{i}$ and $L_{i}^{*}$, it is shown intuitively that this paper has more advantage over [19 21] in the convergence 61876040) and Guangdong Basic and Applied Basic Research Foundation(2020A1515010809).

\section{Compliance with ethical standards}

Conflict of interest The authors declare that they have no conflict of interest.

\section{The Biography of Corresponding Author}

Peitao Gao received his M.S. degree in control engineering from Guangdong University of Technology, Guangzhou, P.R. China, in 2020. Now, he is currently working toward a Ph.D. degree in control theory and engineering at Guangdong University of Thchnology. His research interests include analysis and control for nonlinear systems and complex dynamical networks. 


\section{References}

[1] Choi, J.Y., Farrell, J.A.: Adaptive observer backstepping control using neural networks. IEEE Trans. Neural Netw. Learn. Syst. 12(5), 1103-1112 (2001)

[2] Zhao, D., Wang, Z., Chen, Y., Wei, G.: Proportional-integral observer design for multi-delayed sensor-saturated recurrent neural networks: A dynamic event-triggered protocol. IEEE T. Cybern. 50(11), 4619-4632 (2020)

[3] Rao, H., Liu, F., Peng, H., Xu, Y., Lu, R.: Observer-based impulsive synchronization for neural networks with uncertain exchanging information. IEEE Trans. Neural Netw. Learn. Syst. 31(10), 3777-3787 (2020)

[4] Pagani, G.A., Aiello, M.: The power grid as a complex network: A survey. Physica A. 392(11), 2688-2700 (2013)

[5] Zhao, L., Lai, Y., Park, K., Ye, N.: Onset of traffic congestion in complex networks. Phys. Rev. E 71(2), 026125 (2005)

[6] Sun, Y., Jiang, C., Wang, Z., Xian, L., Zhang, H.: Bi-directional wireless power transfer for vehicle-to-grid systems. J Power Electron. 18(4), 1190-1200 (2018)

[7] Zhu, H., Luo, H., Peng, H., Li, L., Luo, Q.: Complex networks-based energy-efficient evolution model for wireless sensor networks. Chaos Solitons Fractals 41(4), 1828-1835 (2009)

[8] Gao, Z., Wang, Y., Zhang, L.: Adaptive control of structural balance for complex dynamical networks based on dynamic coupling of nodes. Int. J. Mod. Phys. B 32(4), 1850042 (2018)

[9] Liu, L., Wang, Y., Gao, Z.: Tracking control for the dynamic links of discrete-time complex dynamical network via state observer. Appl. Math. Comput. 369, 124857 (2020)

[10] Wang, Y., Wang, W., Zhang, L.: State synchronization of controlled nodes via the dynamics of links for complex dynamical networks. Neurocomputing. 384, 225-230 (2020)

[11] Zhang, L., Wang, Y., Wang, Q., Lei, Y., Wang, F.: Matrix projective cluster synchronization for arbitrarily coupled networks with different dimensional nodes via nonlinear control. Int. J. Robust Nonlinear Control 29(11), 3650-3665 (2019)

[12] Zhang, L., Wang, Y., Wang, Q.: Synchronization for time-varying complex dynamical networks with different-dimensional nodes and non-dissipative coupling. Commun. Nonlinear Sci Numer. Simul. 24(1-3), 64-74 (2015)

[13] Wang, Y., Fan, Y., Wang, Q., Zhang, Y.: Stabilization and synchronization of complex dynamical networks with different dynamics of nodes via decentralized controllers. IEEE Trans. on Circuits Syst. I: Regul. Pap. 59(8), 1786-1795 (2012) 
[14] Zhang, L., Wang, Y., Wang, Q., Qiao, S., Wang, F.: Generalized projective synchronization for networks with one crucial node and different dimensional nodes via a single controller. Asian J. Control 22(4), 1471-1483 (2020)

[15] Zhao, P., Wang, Y.: Asymptotical stability for complex dynamical networks via link dynamics. Math. Meth. Appl. Sci. 43(15), 8706-8713 (2020)

[16] Liu, L., Wang, Y., Li, X., Gao, Z.: Structural balance for discrete-time complex dynamical network associated with the controlled nodes. Mod. Phys. Lett. B 34(10), 2050098 (2020)

[17] Gao, Z., Wang, Y.: The structural balance analysis of complex dynamical networks based on nodes' dynamical couplings. PloS One 13(1), e0191941 (2018)

[18] Peng, Y., Wang, Y., Liu, L.: Asymptotically tracking control for discrete time-varying link system to structural balance via determined external stimulations. Int. J. Mod. Phys. B 34(17), $2050144(2020)$

[19] Gao, Z., Wang, Y., Xiong, J., Zhang, L., Wang, W.: Robust state observer design for dynamic connection relationships in complex dynamical networks. Int. J. Control Autom. Syst. 17(2), 336-344 (2019)

[20] Gao, Z., Xiong, J., Zhong, J., Liu, F., Liu, Q.: Adaptive state observer design for dynamic links in complex dynamical networks. Comput. Intell. Neurosci. 2020, 8846438 (2020)

[21] Gao, Z., Wang, Y., Xiong, J., Pan, Y., Huang, Y.: Structural balance control of complex dynamical networks based on state observer for dynamic connection relationships. Complexity. 2020, $5075487(2020)$

[22] Barnett, S.: Matrix differential equations and Kronecker products. SIAM J. Appl. Math. 24(1), 1-5 (1973)

[23] Hao, Y., Wang, Q., Duan, Z., Chen, G.: Controllability of Kronecker product networks. Automatica 110, 108597 (2019)

[24] Pan, L., Shao, H., Xi, Y., Li, D.: Bipartite consensus problem on matrix-valued weighted directed networks. Sci. China-Inf. Sci. 64(4), 149204 (2021)

[25] Lan, C., Yang, Y., Li, X., Luo, B., Huan, J.: Learning social circles in ego-networks based on muti-view network structure. IEEE Trans. Knowl. Data Eng. 29(8), 1681-1694 (2017)

[26] Tuan, H.D., Apkarian, P., Narikiyo, T., Yamamoto, Y.: Parameterized linear matrix inequality techniques in fuzzy control system design. IEEE Trans. Fuzzy Syst. 9(2), 324-332 (2001)

[27] VanAntwerp, J.G., Braatz, R.D.: A tutorial on linear and bilinear matrix inequalities. J. Process Control 10(4), 363-385 (2000)

[28] Pagilla, P.R., Siraskar, N.B., Dwivedula, R.V.: Decentralized control of web processing lines. IEEE Trans. Control Syst. Technol. 15(1), 106-117 (2007) 
[41] Lü, J., Chen, G., Zhang, S.: Dynamical analysis of a new chaotic attractor. Int. J. Bifurcation Chaos. 12(5), 1001-1015 (2002) web-winding system. J. Frankl. Inst. 355(12), 5217-5239 (2018)

[30] Chu, X., Nian, X., Xiong, H., Wang, H.: Robust fault estimation and fault tolerant control for three-motor web-winding systems, Int. J. Control 2020, 1-13 (2020)

[31] Zhou, J., Lu, J., Lü, J.: Adaptive synchronization of an uncertain complex dynamical network. IEEE Trans. Autom. Control 51(4), 652-656 (2006)

[32] Zhou, J., Lu, J., Lü, J.: Pinning adaptive synchronization of a general complex dynamical network. Automatica 44(4), 996-1003 (2008)

[33] Yu, W., Chen, G., Lü, J.: On pinning synchronization of complex dynamical networks. Automatica 45(2), 429-435 (2009)

[34] Duan, Z., Chen, G., Huang, L.: Synchronization of weighted networks and complex synchronized regions. Phys. Lett. A 372(21), 3741-3751 (2008)

[35] Liu, C., Duan, Z., Chen, G., Huang, L.: L2 norm performance index of synchronization and LQR control synthesis of complex networks. Automatica 45(8), 1879-1885 (2009)

[36] Feng, Y., Duan, Z., Lv, Y., Ren, W.: Some necessary and sufficient conditions for synchronization of second-order interconnected networks. IEEE T. Cybern. 49(12), 4379-4387 (2018)

[37] Cai, C., Wang, Z., Xu, J., Liu, X., Alsaadi, F.E.: An integrated approach to global synchronization and state estimation for nonlinear singularly perturbed complex networks. IEEE T. Cybern. 45(8), 1597-1609 (2015)

[38] Chen, Y., Wang, Z., Shen, B., Dong, H.: Exponential synchronization for delayed dynamical networks via intermittent control: dealing with actuator saturations. IEEE Trans. Neural Netw. Learn. Syst. 30(4), 1000-1012 (2019)

[39] Wang, J., Wei, P., Wu, H., Huang, T., Xu, M.: Pinning synchronization of complex dynamical networks with multiweights. IEEE Trans. Syst. Man. Cybern. Syst. 49(7), 1357-1370 (2019)

[40] Xing, W., Shi, P., Agarwal, R.K., Zhao, Y.: A survey on global pinning synchronization of complex networks. J. Frankl. Inst. 356(6), 3590-3611 (2019)

[29] Chu, X., Nian, X., Sun, M., Wang, H., Xiong, H.: Robust observer design for multi-motor 
Figures

\section{Node $k$}

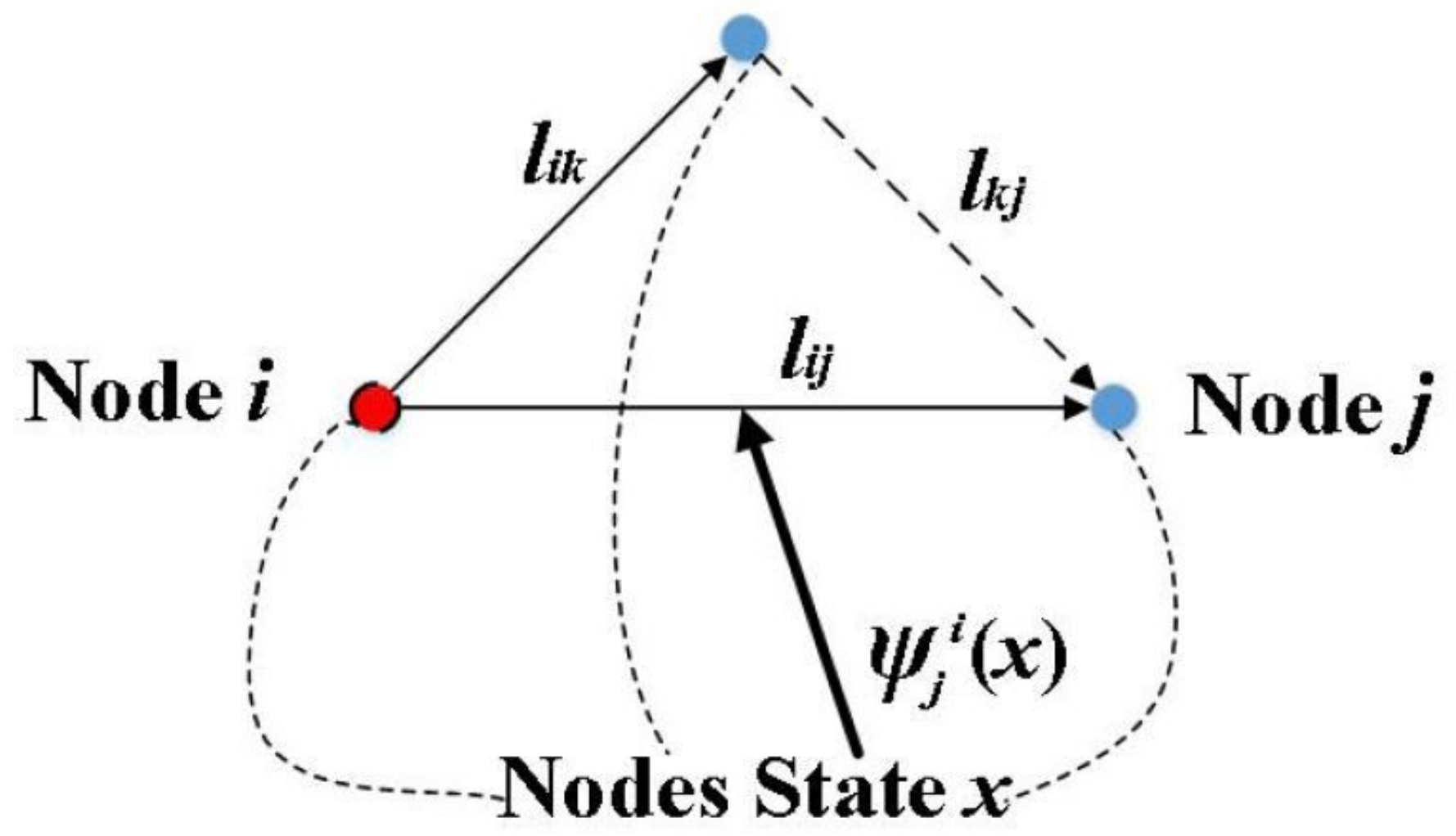

Figure 1

The schematic diagram of lij influenced by lik and the coupling vector $\psi \mathrm{i} j(\mathrm{x})$ in Equation (4)

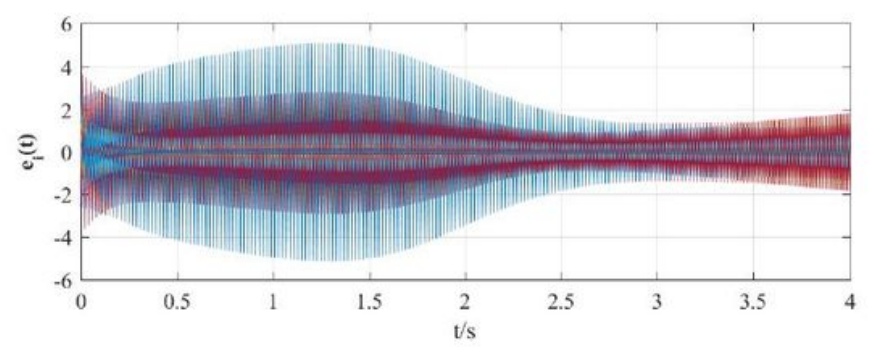

(a)

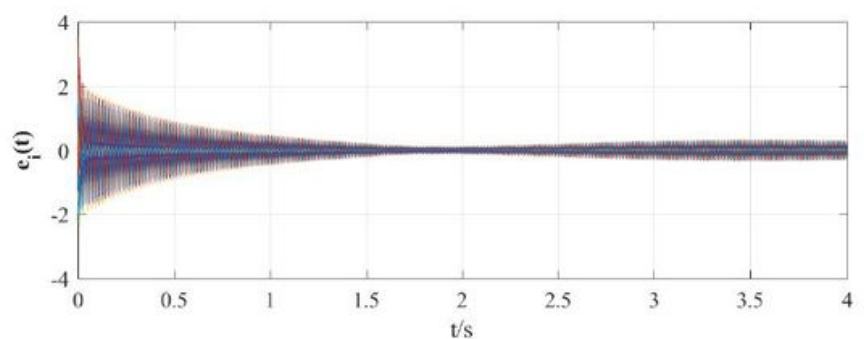

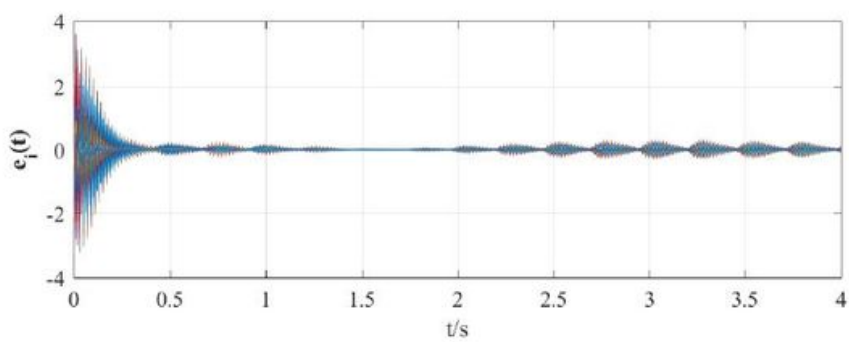

(b)

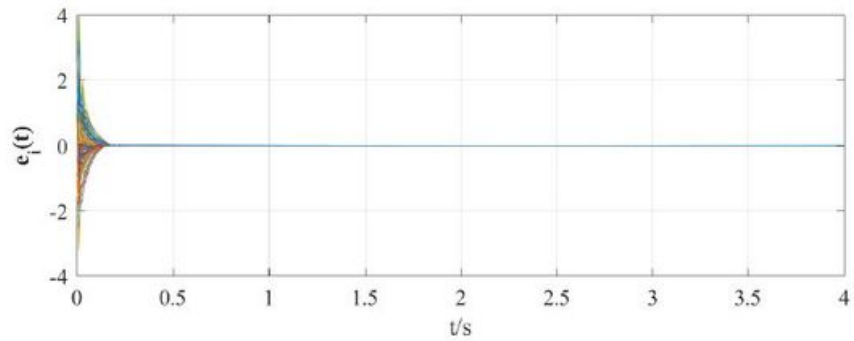

(c) 
Figure 2

(a).The tracking error curves of NS with the controller in [19]; (b). The tracking error curves of NS with the controller in [20]; (c).The tracking error curves of NS with the controller in [21]; (d). The tracking error curves of NS with the controller in this paper
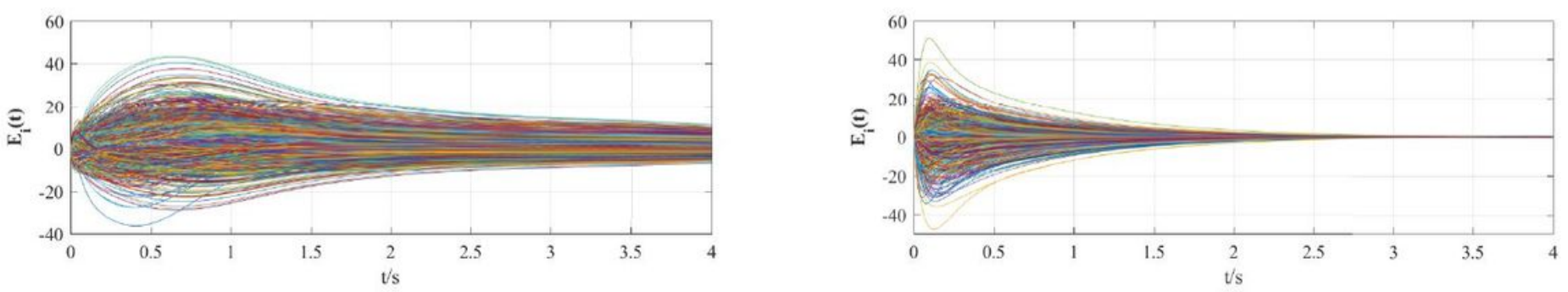

(a)
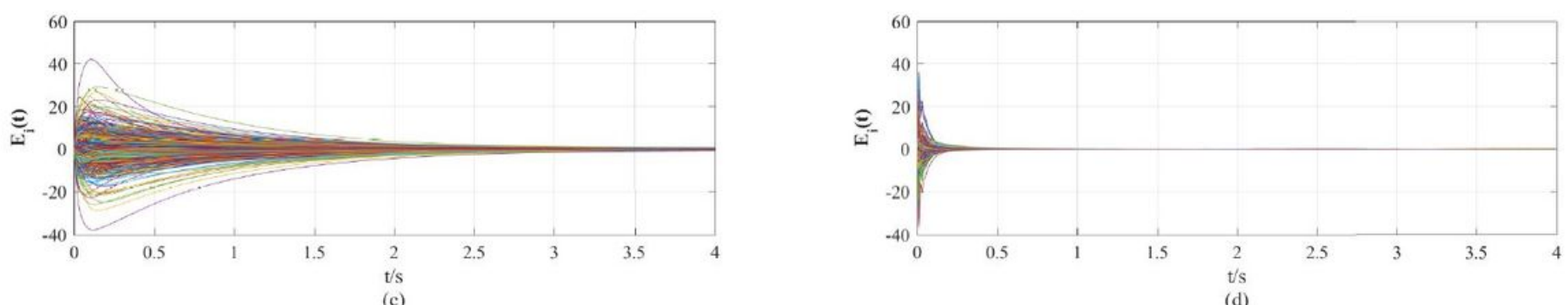

\section{Figure 3}

(a).The tracking error curves of OLS with the controller in [19]; (b).The tracking error curves of OLS with the controller in [20]; (c).The tracking error curves of OLS with the controller in [21]; (d). The tracking error curves of OLS with the controller in this paper 


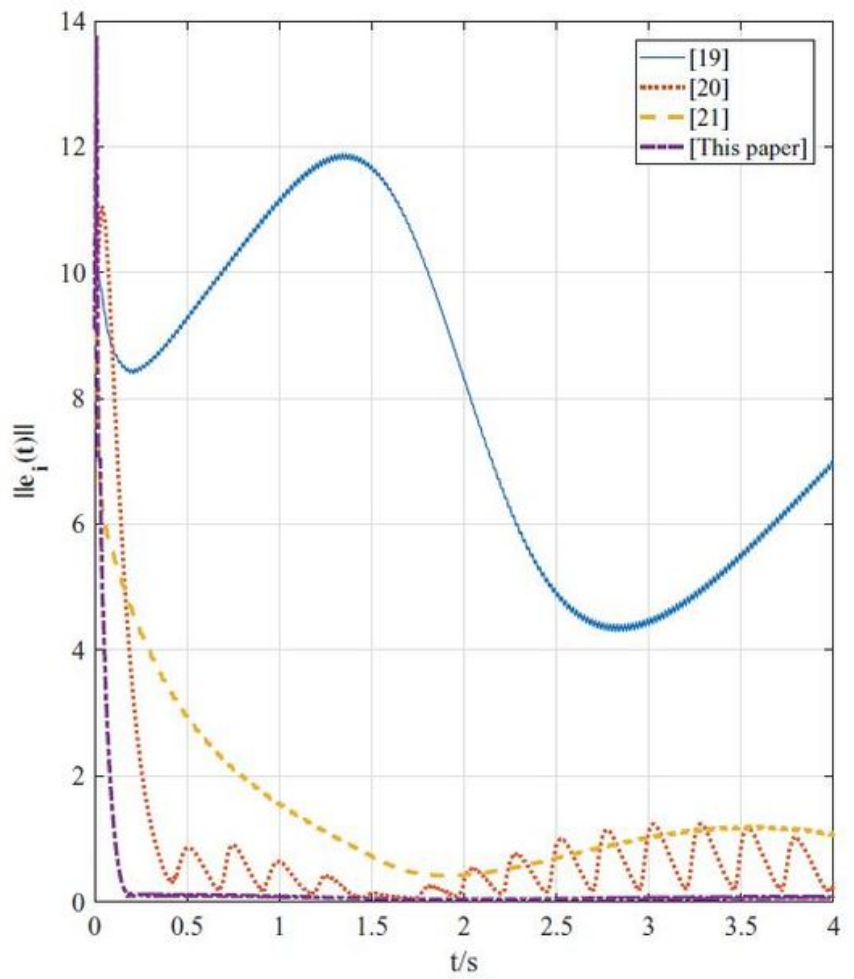

(a)

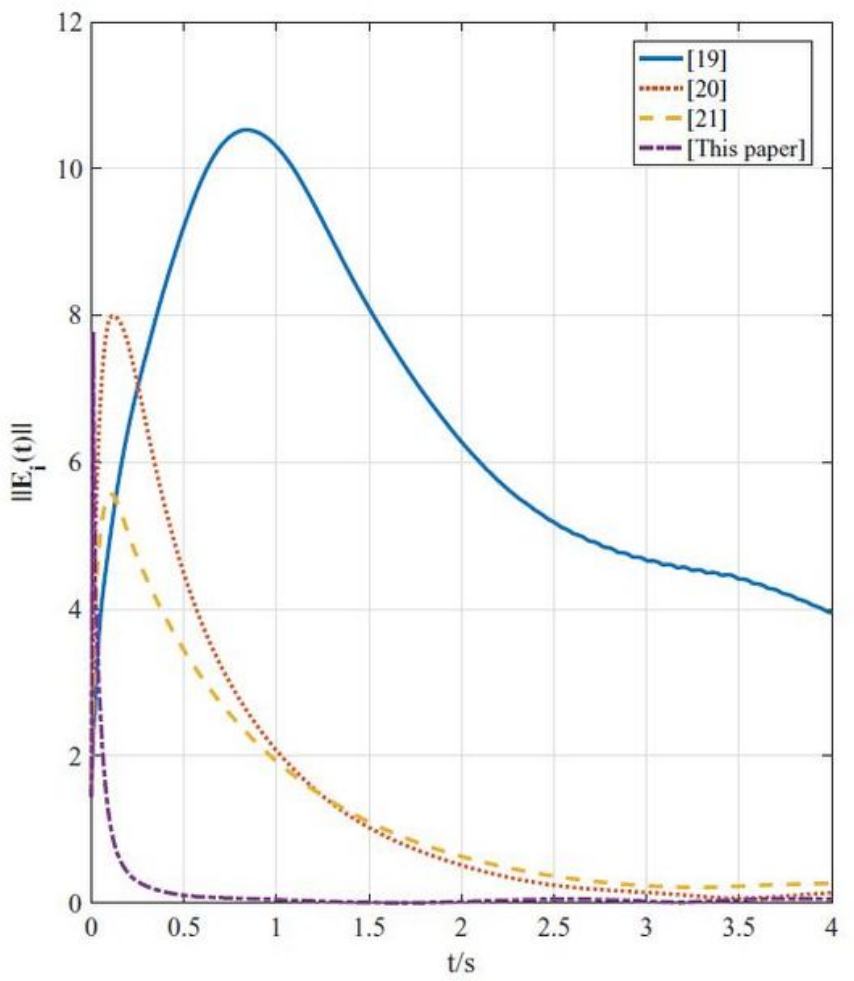

(b)

\section{Figure 4}

(a).The curves of tracking error norm $₫ e i \rrbracket$ of NS with the controller in [19-21] and this paper; (b).The curves of tracking error norm $\triangle \mathrm{E}$ \ of OLS with the controller in [19-21] and this paper 


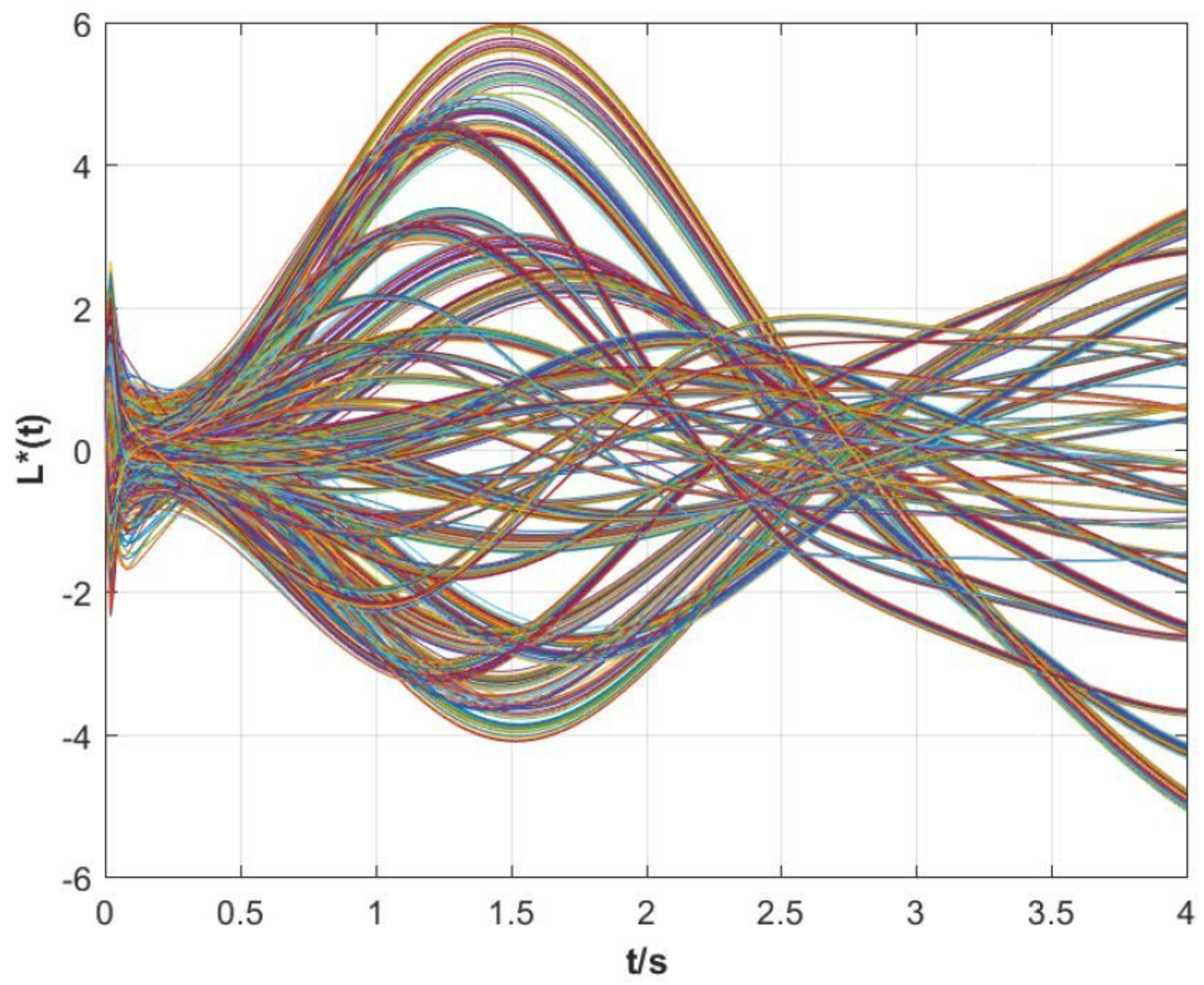

Figure 5

The orbit curves of $L \otimes i s$ in this paper 